\title{
COMPOSICIÓN FISICOQUIMICA DE LA CARNE DE OVEJO, POLLO, RES Y CERDO
}

\author{
PHYSICOCHEMICAL COMPOSITION OF MEAT OF SHEEP, CHICKEN, RES AND PORK
}

\author{
*1León C. Mariana; ${ }^{1}$ Orduz C. Ana; ${ }^{1}$ Velandia C. Magaly
}

${ }^{1}$ Universidad de Pamplona, Facultad de Ingenierías y Arquitecturas Programa de Ingenierías de Alimentos, Pamplona, Norte de Santander - Colombia. Correo electronico:carito mcl@hotmail.com

Recibido: Junio 20 de 2017; Aceptado 30 de noviembre de 2017

\section{RESUMEN}

Características como color, $\mathrm{pH}$, capacidad de retención de agua y poder de emulsificación son importantes en productos primarios como la carne, ya que a partir de estas se puede establecer su destino, bien sea para el consumo directo $y / 0$ transformación, por ello esta investigación se realizó con el fin de evaluar características fisicoquímicas y tecnológicas de cuatro tipos de carne; el método colorimétrico y el de espectrometría de absorción; los resultados fueron evaluados estadísticamente empleando la técnica de varianza con el programa SPSS21. Del estudio se encontró que las carnes de pollo, cerdo y ovejo presento $\mathrm{pH}<6,0$ característico del tipo DFD con una baja capacidad de retención de agua (CRA), la carne de cerdo presentó mayor contenido de proteína. De las 
Universidad de Pamplona

Autor a quien dirigir la correspondencias: León C. Mariana Correo carito mcl@hotmail.com muestras analizadas la carne de res presento menor luminosidad, relacionado con su baja coloración roja y amarronamiento debido a la presencia de Meta mioglobina. Las carnes presentaron un promedio de humedad del $75 \%$ el cuál se encuentra en los rangos establecidos, concluyendo que las propiedades fisicoquímicas como el color y la mioglobina se encuentran íntimamente relacionadas.

Palabras clave: Carne, composición fisicoquímica, espectrofotometría, mioglobina, proteína.

\section{SUMMARY}

Characteristics such as color, $\mathrm{pH}$, water retention capacity and emulsification power are important in primary products such as meat, since from these can be established their destination, either for direct consumption and / or transformation, so this research was conducted in order to evaluate physicochemical and technological characteristics of four types of meat; the colorimetric method and the absorption spectrometry method; the results were statistically evaluated using the variance technique with the SPSS21 program. From the study it was found that chicken, pork and sheep meat had $\mathrm{PH}<6.0$ characteristic of the DFD type with a low water retention capacity (CRA), the pork meat had a higher protein content. Of the samples analyzed, the beef presented less luminosity, related to its low red coloration and browning due to the presence of Meta myoglobin. The meats presented an average humidity of $75 \%$ which is in the established ranges, concluding that the physicochemical properties such as color and myoglobin are closely related.

Key words: Meat, Physicochemical composition, Spectrophotometry, Myoglobin, Protein. 


\section{INTRODUCCIÓN}

El pH es uno de los principales parámetros a considerar para verificar la calidad de la carne, porque afecta varias de sus cualidades (color, capacidad de retención de agua, etc.). El pH es definido como el logaritmo negativo de la concentración de protones. Tiene una escala entre 0 y 14 . Un valor de $\mathrm{pH}$ por debajo de 7 es considerado como ácido, y por encima de un valor de 7 se considera alcalino o también denominado básico.

Este valor se disminuye tras la muerte del animal, principalmente, debido a la degradación del glucógeno a ácido láctico, una reacción en la que el músculo trata de producir energía en ausencia de oxígeno. Esta reacción, depende importantemente de la actividad de una serie de enzimas que son sensibles a la temperatura, por lo que es relevante considerar la temperatura del músculo al momento de hacer la medición del pH. El pH del músculo de animales sanos y vivos es de alrededor de 7.04 (Johnson, 1994).

Las emulsiones cárnicas constituyen un sistema de dos fases, aunque no son sistema de emulsión propiamente dicho debido a que la fase dispersa se encuentra en glóbulos de más de cinco micras. La capacidad de emulsificación (CE) es la habilidad de la carne de sostener la grasa y producir emulsiones estables. Las carnes más apropiadas para formar emulsión son aquellas que poseen elevado contenido de proteínas contráctiles. Estas proteínas recubren o envuelven los glóbulos de grasa y al someter la emulsión a la acción del calor coagulan formando una especie de matriz rígida que atrapa cada partícula grasa. Si la cantidad de proteínas contráctiles es pequeña, en relación a la superficie de grasa a cubrir, los glóbulos grasos no cubiertos, o parcialmente envueltos, se separan de la emulsión en la etapa de calentamiento y se rompe la emulsión. En formulaciones pobres de emulsiones cárnicas, es decir, con exceso de grasa, poca cantidad de fibras musculares y alta proporción de colágeno este último envuelve las partículas grasas, pero al calentar la emulsión el colágeno se transforma en gelatina, liberándose la grasa líquida que se separa. (Medina L., A, 2009)

Las proteínas son polímeros de aminoácidos que se unen entre sí por enlaces peptídicos (aminas). Cada especie animal e incluso cada tejido tienen sus propias proteínas características, la mayor parte de las cuales son materias constitutivas de los tejidos blandos del organismo y otras desempeñan su misión como enzimas, que catalizan todos los procesos bioquímicos. Las proteínas de 
la carne, se caracterizan por tener un alta valor biológico, lo que implica una muy adecuada proporción entre los aminoácidos que la conforman ya que proporciona todos los aminoácidos esenciales en cantidades equivalentes a los requerimientos del humano. Es una proteína altamente digestible y fácilmente absorbible. E contenido de proteína de la carne cruda es aproximadamente de $19-23 \%$, éste varía inversamente proporcional a la grasa y debido a las pérdidas de humedad y grasa durante el cocinado; la proteína de la carne cocinada aumenta a $25-30 \%$. Todos los aminoácidos que constituyen las proteínas contienen nitrógeno en su molécula, ésta es una característica que permite determinar el contenido de proteína a partir de la cuantificación de este elemento. Sin embargo, la cantidad de nitrógeno presente en cada aminoácido, es variable. Por ejemplo, el porcentaje en peso de nitrógeno en una molécula de tirosina, es de $8.6 \%$; mientras que en la arginina es de $35.9 \%$ (Hui et al., 2001).

Desde 1880, Johan Kjeldahl propuso el método para la determinación de proteína cruda. Un método que se sustenta en la cuantificación de nitrógeno en una muestra y en el cual se acepta que no necesariamente todo el nitrógeno determinado se refiere al nitrógeno a del grupo amino de los aminoácidos o nitrógeno proteico, ya que la determinación puede incluir el nitrógeno no proteico de amidas, ácidos nucléicos y aminoácidos libres. El método Kjeldahl se basa en la destrucción de la materia orgánica con ácido sulfúrico concentrado, formándose sulfato de amonio, que en exceso de hidróxido de sodio libera amoníaco, el cual se destila recibiéndolo en ácido bórico, formándose borato de amonio, que se valora con ácido clorhídrico. Este método está basado en tres fases: digestión, destilación y titulación.

Durante la digestión se busca la conversión a amonio (NH4+) de todos los compuestos nitrogenados presentes en la muestra.

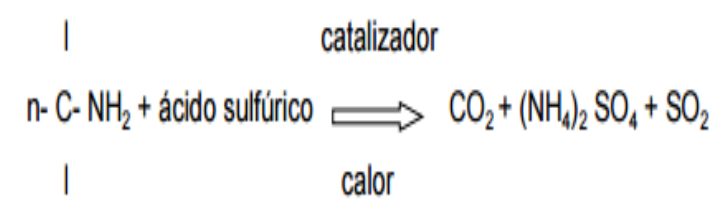

Al completarse la digestión, la mezcla se alcaliniza con una solución de $\mathrm{NaOH}$, con el propósito de liberar el $\mathrm{NH} 3$ a partir del $\mathrm{NH} 4$ por arrastre de vapor hacia una solución que contiene ácido bórico, y de esa manera fijarlo para un análisis posterior.

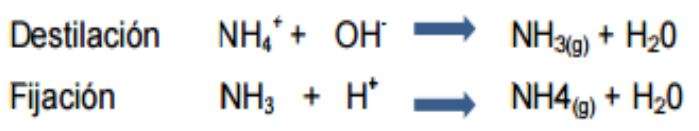


El amonio fijado en la solución de ácido bórico se cuantifica por titulación con una solución estándar de ácido clorhídrico $(0.1 \mathrm{~N})$, formando un complejo estable, y pudiendo observarse debido al cambio de color que experimenta la solución de ácido bórico (rojo a amarillo), producido por el amoníaco, y que alcaliniza la solución progresivamente a medida que es captado por el ácido bórico. Esto es visible gracias al indicador rojo de metilo; aunque es posible usar otro indicador según el rango de viraje. (Nielsen, 2010).

La carne contiene aproximadamente entre un 70 y $75 \%$ de agua, de la cual el $70 \%$ es agua libre que se encuentra entre los espacios de los filamentos de actina $y$ miosina, el otro $5 \%$ es agua ligada a proteínas. Cuando se hace la determinación de humedad principalmente lo que se mide es el agua libre.

El análisis del contenido de humedad o de materia seca, es en el análisis bromatológico probablemente el más frecuentemente realizado, debido a que permite conocer el grado de dilución de los nutrimentos o componentes de la muestra (Bradley, 2003).

La capacidad de retención de agua se puede definir como la aptitud de la carne para mantener ligada su propia agua, incluso bajo la influencia de fuerzas externas (presión, calor, etc.), o también como la aptitud para fijar agua añadida. Muchas de las propiedades sensoriales de la carne como son el color, la textura y la firmeza, están relacionadas con la cantidad de agua que se tiene contenida o retenida en la carne. Nutricionalmente, una baja CRA resulta en pérdidas importantes de agua, que acarrean, proteínas, minerales y vitaminas hidrosolubles. Desde el punto de vista industrial, la capacidad de una carne para retener el agua originalmente contenida, así como el agua que se añada durante los procesos industriales, por ejemplo durante el marinado o la inyección, influye en la eficiencia del sistema y dicta en parte el rendimiento final del producto. Una pobre retención de agua, provoca un goteo constante que interfiere en los sistemas de empaque, así como en los sistemas de salazón en seco. (Swatland, 1991).

La CRA es influenciada (hasta cierto punto) por el pH del músculo, mientras más alejado este el $\mathrm{pH}$ del punto isoeléctrico de las proteínas del músculo, más agua se retendrá. Por ejemplo, en valores superiores a 5.8 de $\mathrm{pH}$, se favorece la capacidad de las proteínas para ligar las moléculas de agua. Además del $\mathrm{pH}$, otros factores que afectan la CRA, son la especie de que proviene la carne, el tipo de fibra, la estabilidad oxidativa de sus membranas, el proceso de maduración, y de ser el caso, el sistema 
utilizado para congelar y descongelar las carnes (Trujillo et al., 2014; Trujillo et al., 2016).

La medida de la capacidad de retención de agua por la carne mediante el control del fluido liberado al aplicar presiones externas (deformando la muestra), ha venido utilizándose ampliamente. De los métodos de deformación de la carne tras la aplicación de altas presiones, uno de los más utilizados dada su sencillez, es el de compresión sobre papel filtro.

La versión original de este método la desarrollaron Grau y Hamm en 1953 y 1957 (Hamm, 1986), partiendo de asumir de que el área del papel mojado por el jugo que queda fuera de la carne, es proporcional al agua liberada, y que la presión ejercida comprimiendo a mano las placas, es tan grande que las diferencias de presión no afectan a dicha área. Desde entonces, se han empleado múltiples versiones que varían el peso de la muestra, su estado, la presión a ejercer, o la forma de expresar el resultado (Cañeque y Sañudo, 2005).
\% Jugo liberado = $($ Peso final del papel filtroPeso Inicial del papel filtro) / Peso de muestra *100

El color de la carne fresca es el principal atributo que influye en la decisión de compra, dado que el consumidor asocia el color con el grado de frescura y calidad (Brewer et al., 2002).

En la carne, al igual que otros materiales no metálicos, al incidir un rayo de luz en su superficie se produce una reflexión difusa, esa reflexión es lo que se define como el color. Así, al incidir una luz blanca sobre una substancia, ciertas longitudes de onda que componen esa luz blanca, serán absorbidas por la muestra, el color estará formado por la combinación de aquellas longitudes de onda que no fueron absorbidas por la substancia.

El color percibido ha sido definido por CIE (Commision Internationale de L'Eclairage) como el atributo visual que se compone de una combinación cualquiera de componentes cromáticos y acromáticos (Alberti et al., 2005).

\section{MATERIALES Y MÉTODOS}

Se realizó la compra de 300 gramos de lomo de carne de res, que fue adquirida en charcutería "Carnes el humilladero" de la ciudad de Pamplona, Norte de Santander.
Las pruebas fisicoquímicas en carne se llevaron a cabo en la Universidad de Pamplona Colombia, sede pamplona, ubicada en el bloque edificio Antonio José 
Betancourt Walker (AJ), Centro Tecnológico de Alimentos (CETA).

Determinación de pH. Según el método empleado por la A.O.A.C 981.12 se pesaron 10 gramos de carne, se transfirieron a un vaso de licuadora y se adicionaron $100 \mathrm{ml}$ de agua homogeneizando durante 1 minuto. Filtrando el homogeneizado con gasa. Tomando lectura del $\mathrm{pH}$ introduciendo el electrodo del potenciómetro realizado por duplicado.

\section{Determinación de acidez total titulable.} Según el método de la A.O.A.C 900.02 se pesaron 10 gramos de carne, se transfirieron a un vaso de licuadora y se adicionaron $100 \mathrm{ml}$ de agua homogeneizando durante 1 minuto. Filtrando el homogeneizado con gasa, pasándolo a un matraz aforado de 250 $\mathrm{mL}$ aforando con agua destilada. Se pasó 25 $\mathrm{mL}$ del filtrado a un Erlenmeyer, se añadió 75 $\mathrm{mL}$ de agua destilada y 2 gotas de fenolftaleína, se agito suavemente y se tituló con $\mathrm{NaOH}$.1N. Se preparó una muestra en blanco con agua destilada. Se realizó el reportaje en porcentaje de ácido láctico aplicando la siguiente fórmula:

$\%$ ácido láctico $=\frac{(V-V b) *\left(N_{N a O H}\right) *(f d)}{\text { peso de la muestra }}$

Donde

$V=$ volumen de $\mathrm{NaOH}$ gastado en la muestra
$V b=$ volumen de $\mathrm{NaOH}$ gastado en el blanco

$N=$ normalidad del $\mathrm{NaOH}$

$f d=$ factor de disolución

Capacidad de emulsificación (CE). Se homogeneizo $25 \mathrm{~g}$ de carne con $100 \mathrm{~mL}$ de solución fría de $\mathrm{NaCl} 1 \mathrm{M}$. Se tomó $12.5 \mathrm{~g}$ del homogeneizado y e añadió $37.5 \mathrm{~mL}$ de solución fría de $\mathrm{NaCl} 1 \mathrm{M}$, se mezcló por 3 minutos a baja velocidad. Sin apagar la licuadora o el homogeneizador, se añadió $50 \mathrm{~mL}$ de aceite y se esperó a que se formara la emulsión. Con ayuda de una bureta y sin detener el mezclado, se adiciono en forma continua más aceite de maíz hasta la ruptura de la emulsión. Se realizó esta determinación por duplicado y se reportó la cantidad de aceite emulsionado por $g$ de muestra.

Determinación de proteína. Se determinó la cantidad de proteína por el método de Kjeldahl según norma ISO 937 de 1978. Para ello se empleó una unidad de digestión K424 de seis puestos y una unidad de destilación K355 de (marca Büchi). Se pesó 0,5 g. de carne y se dispuso en un tubo, $0,5 \mathrm{~g}$ sulfato cúprico, $10 \mathrm{~g}$. de Sulfato de potasio. Se agregó $10 \mathrm{ml}$ ácido sulfúrico y se dispuso el digestor. Se dejó en el digestor por una hora. Se dejó enfriar el tubo y se agregó $30 \mathrm{ml}$ de agua, y se dispuso en la unidad destilación. Luego se tomó un Erlenmeyer al cual se agregó 3 gotas de indicador, se dispuso en 
la unidad se agregó $100 \mathrm{ml}$ de ácido Bórico al $2 \%$, luego se agregó Hidróxido de Sodio. Se dio inicio a la destilación. De la muestra obtenida, se obtuvo $10 \mathrm{ml}$ y se tituló con ácido clorhídrico $0,1 \mathrm{~N}$ hasta viraje a rosa.

$\%$ proteina $=\frac{14 * N * V * 100 * \text { factor }}{m * 1000}$

Donde:

$V$ : volumen gastado de $H C L$

m: masa de la muestra

factor: 6,25

$N$ : normalidad 0,1

Humedad. Según norma A.OA.C de 1990 se determinó por gravimetría, con la balanza de humedad Ohaus M 35, trabajando $3 \mathrm{~g}$. de muestra de carne homogenizada, la cual fue sometida a una temperatura de $110^{\circ} \mathrm{C} \pm 1$ durante $55 \mathrm{~min}$, reportándose como porcentaje de humedad.

\section{Capacidad de retención de Agua (CRA).}

Se determinó por el método propuesto por Grau et al 1953, donde se tomaron 0,30g de carne picada, libre de grasa y tejido conjuntivo. Se Dispuso la muestra entre dos papeles de filtro Whatman no1 de $55 \mathrm{~mm}$ de diámetro, situados cada uno en una caja de petri los cuales se presionaron con un peso de $5 \mathrm{~kg}$ por 5 minutos. Posteriormente, y tomando el papel filtro con la muestra de carne adherida, se midió la superficie de la mancha del agua expulsada y la de la carne con un pie de rey, y por diferencia entre el valor de ambas superficies, se obtuvo el valor del anillo del líquido (RZ).

Determinación del color mediante espectrometría de absorción. Según el protocolo descrito por la norma NTE INEN 2682:2013. Se homogeneizo $10 \mathrm{~g}$ de carne con $90 \mathrm{~mL}$ de agua destilada, se filtró primero a través de gasa y posteriormente a través de papel filtro Whatman del No. 1 se colocó el filtrado en una celda de espectrofotómetro, se obtuvo el espectro de absorbancia entre los 480 a $650 \mathrm{~nm}$. Se obtuvo los valores de absorbancia a 503, 525, 557 y $582 \mathrm{~nm}$, se empleó agua destilada como blanco. 


\section{RESULTADOS Y DISCUSIÓN.}

Los valores de acidez titulable de las carnes obtenidos en el presente trabajo (Tabla 1) están por debajo por los valores reportados por (Solis, 2005) de 0,30 a 0,60 por cada 100 $g$ de carne, esta condición puede presentarse en canales de carne más oscura y más seca de lo normal, y tiene una textura más firme. Por consiguiente, hay poca generación de ácido láctico luego del sacrificio, produciéndose así una carne DFD. La carne con esta condición implica que la canal procedió de un animal estresado lesionado o enfermo antes de su sacrificio.

Tabla 1. Resultados fisicoquímicos y comparación entre los tipos de carne

\begin{tabular}{|l|l|l|l|l|l|l|}
\hline $\begin{array}{l}\text { Ti } \\
\text { po } \\
\text { de } \\
\text { ca } \\
\text { rn } \\
\text { e }\end{array}$ & pH & $\begin{array}{l}\text { Acid } \\
\text { ez }\end{array}$ & CRA & CE & $\begin{array}{l}\text { Prote } \\
\text { ina }\end{array}$ & $\begin{array}{l}\text { Hum } \\
\text { edad }\end{array}$ \\
\hline PO & 6,65 & 0,28 & 17,60 & 2,83 & 17,85 & 77,3 \\
LL & $5 \pm, 0$ & $3 \pm, 0$ & $3 \pm 2,5$ & $4 \pm 2$, & $0 \pm, 49$ & $75 \pm$, \\
O & $07^{\mathrm{a}}$ & $06^{\mathrm{a}}$ & $87^{\mathrm{a}}$ & $58^{\mathrm{a}}$ & $49^{\mathrm{a}}$ & $487^{\mathrm{a}}$ \\
\hline OV & 6,45 & 0,13 & 12,96 & 1,35 & 18,90 & 77,2 \\
$\mathrm{EJ}$ & $5 \pm, 0$ & $4 \pm, 0$ & $7 \pm 1,3$ & $1 \pm 1$, & $0 \pm, 49$ & $05 \pm$, \\
$\mathrm{O}$ & $07^{\mathrm{b}}$ & $00^{\mathrm{b}}$ & $51^{\mathrm{ab}}$ & $35^{\mathrm{a}}$ & $49^{\mathrm{a}}$ & $304^{\mathrm{a}}$ \\
\hline $\mathrm{RE}$ & 5,47 & 0,27 & 16,69 & 1,75 & 23,97 & 71,0 \\
$\mathrm{~S}$ & $5 \pm 0$ & $3 \pm, 0$ & $9 \pm 1,7$ & $6 \pm 1$, & $5 \pm, 49$ & $30 \pm 1$ \\
& $21^{\mathrm{c}}$ & $01^{\mathrm{a}}$ & $56^{\mathrm{a}}$ & $75^{\mathrm{b}}$ & $49^{\mathrm{b}}$ &, $41^{\mathrm{b}}$ \\
\hline $\mathrm{CE}$ & 6,30 & 0,15 & 23,14 & 4,79 & 30,85 & 74,9 \\
$\mathrm{RD}$ & $5 \pm, 0$ & $1 \pm, 0$ & $4 \pm 4,7$ & $4 \pm 4$, & $4 \pm 2,5$ & $50 \pm$, \\
$\mathrm{O}$ & $07^{\mathrm{d}}$ & $07^{\mathrm{c}}$ & $94^{\mathrm{ac}}$ & $79^{\mathrm{c}}$ & $68^{\mathrm{c}}$ & $28^{\mathrm{c}}$ \\
\hline
\end{tabular}

Los $\mathrm{pH}$ obtenidos en las diferentes carnes (tabla 1), reflejan que los valores de las muestras de carne de pollo, ovejo y cerdo pertenecen a carnes DFD, oscura, firme y secas (Zimerman, 2008), mientras que la carne de res presentó un valor inferior a 6 , lo que la convierte en una carne PSE (pálida, suave, exudativa), las carnes DFD se caracterizan por su capacidad para la retención de agua según (Hernández, Aquino, \& Ríos, 2013), la carne de res arrojo un $\mathrm{pH}$ bajo lo que provoco la desnaturalización de las proteínas y a una glucogenólisis rápida genera una carne (PSE) y la lenta genera una carne (DFD).

El valor del porcentaje de jugo extraído de las carnes se expresa en la tabla 1, obteniendo como resultado de la práctica que la carne de cerdo $23,144 \pm 4,794$, presento la menor capacidad de retención de agua frente a las demás, por otra parte, la carne de ovino exhibo el menor porcentaje en jugo extraído $12,967 \pm 1,35$, esto quiere decir que la carne de ovejo es la carne con mayor capacidad para ligar su propia agua al someterse a diversos procesos en los que se involucre variables externas como calor y presión, esta mantendrá sus atributos de calidad como terneza, jugosidad y flavor, características que predominan en las carnes con un porcentaje de CRA normales, como lo arrojaron las muestras de pollo, ovejo y res. (Leal \& L Jiménez, 2015) 
La capacidad emulsionante en la carne del cerdo es mayor comparada con las demás carnes (pollo, res y ovejo) como se puede observar en la tabla 1, lo que indica que la carne de cerdo presenta una mayor capacidad emulsionante de las proteínas para ligar grasas, esto nos permite desarrollar diferentes aplicaciones a nivel industrial. (J. Ruíz, 2015).

En la práctica de laboratorio se obtuvo el mayor porcentaje de proteína en la carne de cerdo y la carne de res, los valores obtenidos superan los obtenidos por (Solis, 2005), los cuales se encontraban en $21,8 \%$ para res y $21,9 \%$ para cerdos, los resultados de proteína en pollo y ovejo se encuentran en los rangos normales. La proteína es el componente mayoritario en las carnes y su concentración depende de la raza, alimentación y edad.

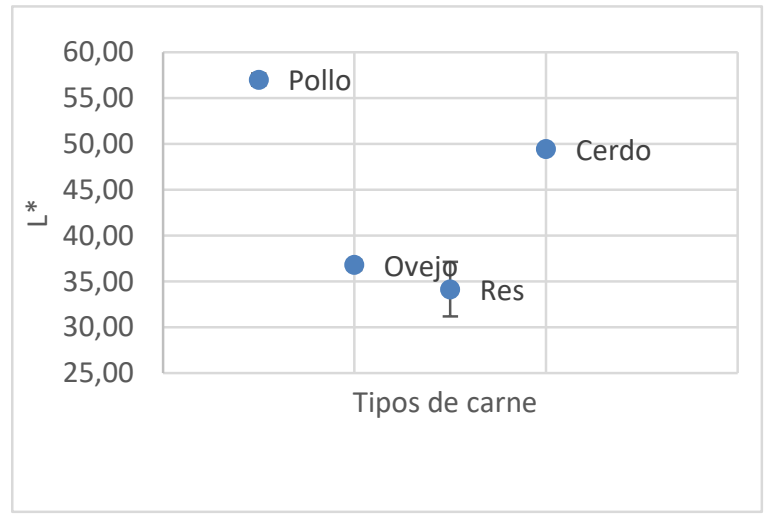

$\rho$-Valor $\leq 0,05$ error típico significativo.

Figura 1. Luminosidad en los diferentes tipos de carne.
En la figura 1, se evidencian que la carne con menor luminosidad es la res, datos que a su vez representan el mayor grado o porcentaje de error, estos valores de color son complementados con el espacio cromático $a^{*}$ y $b^{*}$ los cuales se relacionan en la siguiente figura.



$\rho$-Valor $\leq 0,05$ error típico significativo.

Figura 2. Espacio cromático a y b en los diferentes tipos de carne.

Los valores obtenidos en la res, evidencian que hay una baja tinción roja, analizado a partir del valor de $a^{*}=8,9950 \pm 0,1626$ él cual es inferior al arrojado por la carne de cerdo y ovejo, como también el valor de b* el cual corresponde a 7,7100 $\pm 0,2121$ siendo el valor más inferior, corroborando la poca luminosidad representada en la (figura 1), la cuál puede ser relacionado a su vez con las bajas tonalidades amarillas que corresponde al veteado de la grasa intramuscular, debido a que la parte analizada era bastante magra. 
Tabla 2. Resultados de color por espectrometría

\begin{tabular}{|l|l|}
\hline Aspecto evaluado & Valor \\
\hline Mioglobina & $0,13645 \pm 0,0005$ \\
\hline Oximioglobina & $0,07023 \pm 0,0014$ \\
\hline Metamioglobina & $0,51914 \pm 0,0002$ \\
\hline
\end{tabular}

El color en particular está fuertemente asociado con la calidad de carne esperada. El contenido de mioglobina y su estado de oxidación en la superficie son los principales factores que determinan el color de la carne. La mioglobina es una proteína soluble en agua que contiene hierro en su estructura. En la carne fresca se encuentra principalmente en tres estados básicos: desoximioglobina (DMb), oxiomioglobina $(\mathrm{OMb})$ y metamioglobina $(\mathrm{MMb})$, (Mancini y Hunt 2005). Los colores característicos de estas tres formas de mioglobina son consecuencia de sus espectros de reflectancia distintivos.

En la superficie de la carne, estas tres formas se encuentran en un equilibrio dinámico impulsado por la disponibilidad de oxígeno. En ausencia de oxígeno, la desoxiomioglobina es la forma dominante, que imparte un color rojo violáceo típicamente asociado con carne envasada al vacío o con trozos frescos inmediatamente después del corte. Cuando la carne se expone al oxígeno se forma el pigmento oxigenado de la forma oximioglobina, acompañado por el desarrollo de un color rojo cereza brillante.

Los resultados correspondientes a las figuras 2 y 1 , se corroboran con la valoración por espectrofotometría en donde se presentó que la carne de res es menos roja y amarilla en comparación con las otras especies, y lo que por observación directa se percibió como un color rojo amarronado, los análisis la tabla 1 , de los pigmentos, relacionan directamente el color de la superficie de la carne y este último permite verificar el estado de la mioglobina presente en la carne, en donde el dato más relevante fue el de la metamiogobina con un valor de 0,51914 \pm 0,0002 lo cual muestra un deterioro del color en la superficie de la carne que se debe al estado de mioglobina oxidada. A medida que la proporción de metamioglobina aumenta, el color de la carne se vuelve marrón. (Hernández, Sáenz, \& Diñeiro, 2016). 


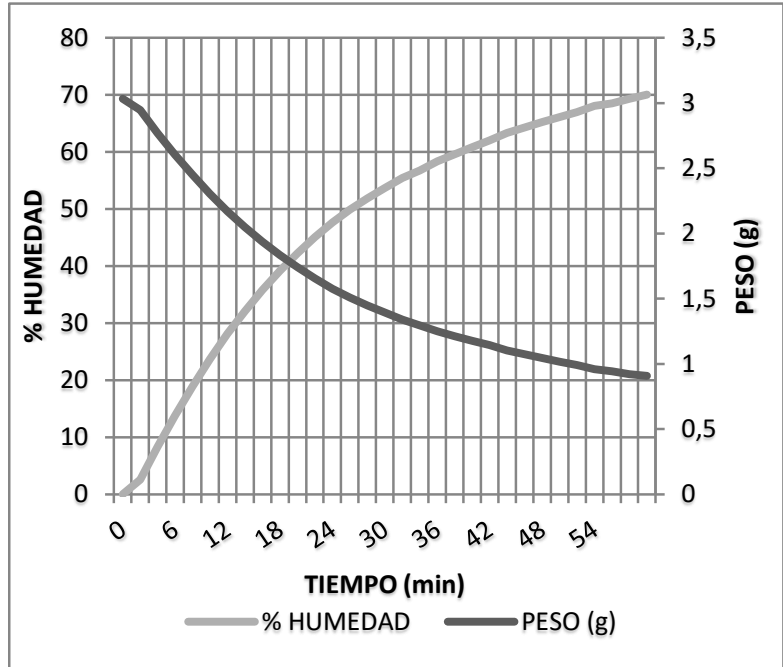

Figura 3. Relación de humedad y peso Vs tiempo en la carne de cerdo.

La figura 3, muestra que la carne de res presentó un porcentaje de humedad promedio del $71 \%$ en comparación con la carne de pollo y ovejo que exhibieron un porcentaje de humedad aproximadamente del $77 \%$, un porcentaje de humedad alto comparado con los resultados de (López Palacios, Rubio Lozano, \& Valdés Martínez, 2000), que exhibe un $72,5 \%$ - $75 \%$, un porcentaje de humedad alto se atribuye a animales menores de dos años, el porcentaje de humedad en bovino y porcino fueron normales, y podemos decir que las muestras analizadas son carnes que cuentan con características de calidad aceptables en términos de jugosidad, terneza, color y sabor.

\section{CONCLUSIONES}

Según los datos obtenidos de la composición fisicoquímica de la carne, se puede evidenciar que la Res presentó características propias de la una carne tipo PSE, relacionado con el descenso abrupto del $\mathrm{pH}$ en el momento posterior al sacrificio del animal, por ende los datos obtenido en el color evidenciaron una carne con baja tinción roja y amarronamiento, lo cual también se debió a la oxidación de la mioglobina, dando lugar a la obtención del ion férrico característico de este color marrón oscuro.
Como también se puede destacar que, de las diferentes especies, el cerdo fue quien obtuvo mayor capacidad emulsificante, haciéndola viable para la elaboración de productos de pastas finas como las salchichas, mortadelas entre otros.

La correcta implementación de las pruebas fisicoquímicas permitió determinar la calidad de la carne como CRA, CE, Acidez, PH, humedad y color, que estas a su vez determinan el estado y uso de la carne y en la que los componentes de la carne se pueden ver afectados por las condiciones de 
manipulación, procesamiento o almacenado y el valor nutricional final de esta, así como su durabilidad.

\section{REFERENCIAS BIBLIOGRAFICAS}

Alberti $P$, Panea B, Ripoll G, Sañudo $C$, Olleta JL, Negueruela l, et al. Medición del color. En: Cañeque V, Sañudo C editores. Estandarización de las metodologías para evaluar la calidad del producto (animal vivo, canal, carne y grasa) en los rumiantes. Madrid, España: MICYT-INIA: Ganadera; 2005: (3)216-225

Bradley R. Moisture and total solids analysis. En: Nielsen $S$ editor. Food analysis. 3a ed. New York, USA: Kluwer Academic; 2003.

Brewer SJ, Wilson JE, McKeith F. The effect of pig genetics and palatability, colorant physical characteristics of fresh loin chops. Meat Sci 2002; 61: 249-256.

Cañeque V, Sañudo C. Estandarización de las metodologías para evaluar la calidad del producto (animal vivo, canal, carne y grasa en los rumiantes). Madrid, España: MICYTINIA: Ganadera; 2005; 3.

Hamm R. Functional properties of the myofibrilar system and their measurements. En: Bechtel PJ editor. Muscle as food.
Orlando, Florida, USA: Academic Press; 1986: 135.

Hernández, B., Sáenz, C., \& Diñeiro, J. (2016). CIELAB color coordinates versus relative proportions of myoglobin redox forms in the description of fresh meat appearance. Journal of food science and technology, 4153-4167.

Hernández, J., Aquino, J., \& Ríos, F. (2013). Efecto del manejo pre-mortem en la calidad de la carne. NACAMEH Vol. 7, No. 2, 4164.

J. Ruíz, D. M. (2015). La calidad de la carne en porcino. Tecnología de Alimentos. , Vol (2). 32-48.

Leal, G. J., \& L Jiménez, R. (2015). La capacidad de retención de agua (CRA) de la carne de bovino y posibles genes candidatos. Departamento de Ciencias de la Producción Animal, Facultad de Medicina Veterinaria y de Zootecnia. Universidad.

López Palacios, M. G., Rubio Lozano, M. S., \& Valdés Martínez, S. E. (2000). Efecto del cruzamiento, sexo y dieta en la 
composición química de la carne de ovinos Pelibuey con Rambouillet y Suffolk. Veterinaria México, vol. 31, 11-19.

Martínez P. Cesar, Verhelst S. Adriana L. (2015). Calidad Microbiológica De Carne Bovina en plantas de beneficio. Revista @limentech, Ciencia y Tecnología Alimentaria. ISSN 1692-7125. Volumen 13 No 1. Pp: $72-80$.

Olis, L. (2005). Manual de practicas de tecnologia de carnes. Facultad de ingenieria en industrias alimentarias.Universidad nacional del centro de Perú, 70-78.

Púa R. Amparo L., Navas G. Norleyn M. (2014). Calidad higiénica y determinación de Escherichia coli y Salmonella spp. en carne de cerdo en expendios de Barranquilla. Revista @limentech, Ciencia y Tecnología
Alimentaria. ISSN 1692-7125. Volumen 12 No 1. Pp: $15-22$.

Trujillo, N. Yanine, Ayala M. Magda, Duran O. Daniel S. (2015). Influencia de la edad y el tiempo de madurez en la capacidad de retención de agua (CRA) en la carne de ovino criollo. Revista @limentech, Ciencia y Tecnología Alimentaria. ISSN 1692-7125. Volumen $13 \mathrm{~N}^{\circ} 1$. Pp: $41-47$.

Trujillo, N. Yanine, Duran O. Daniel S. Hernandez L. Anabelly. (2014). Influencia de la castración, ecorregión y época del año en la calidad sensorial de la carne de ovejo. Revista @limentech, Ciencia y Tecnología Alimentaria. ISSN 1692-7125. Volumen $13 \mathrm{~N}^{\circ} 1$. Pp: $41-47$.

Zimerman, M. (2008). 'pH de la carne y factores que lo afectan. Agrociencia, 56-67. 